\title{
Estilos de aprendizaje de estudiantes universitarios principiantes con modalidad virtual
}

\section{Learning styles of beginning university with virtual modalit}

DOI: http://dx.doi.org/10.17981/cultedusoc.10.2.2019.04

Recibido: 28/11/2018 Aceptado: 15/04/2019

Nelson Leal Bolaño

Universidad Nacional Abierta y a Distancia UNAD (Colombia)

nelson.leal@unad.edu.co

Héctor Aguilera Cuenca

Universidad Nacional Abierta y a Distancia UNAD (Colombia)

hector.aguilera@unad.edu.co

Tatiana Egea Arciniegas

Universidad Nacional Abierta y a Distancia UNAD (Colombia)

tatiana.egea@unad.edu.co

Sara Escobar Jurado

Universidad Nacional Abierta y a Distancia UNAD (Colombia)

sara.escobarnad@unad.edu.co

Para citar este artículo:

Leal, N., Aguilera, H., Egea, T. y Escobar, S. (2019). Estilos de aprendizaje de estudiantes universitarios principiantes con modalidad virtual. Cultura, Educación y Sociedad, 10(1). 47-62. DOI: http://dx.doi.org/10.17981/cultedusoc.10.2.2019.04

\section{Resumen}

La investigación tiene como propósito caracterizar los estilos de aprendizaje de estudiantes universitarios principiantes con modalidad virtual, describiendo la preponderancia de cada uno de los estilos propuestos por Felder y Silverman en 1988. En lo metodológico se trata de un estudio mixto de carácter exploratorio-descriptivo donde participaron 5951 estudiantes, adscritos a programas académicos orientados desde la virtualidad. Los principales resultados destacan la predominancia en los estudiantes de estilos de aprendizajes orientados a lo activo, reflexivo y visual. Se concluye en la pertinencia de identificar acciones que atiendan las necesidades de los estudiantes en forma tal que se sientan motivados y fortalezcan su sentido de pertenencia institucional, de tal manera que se apropien de la didáctica de mediación virtual y logren mejorar su desempeño académico.

Palabras clave: Estilos de aprendizaje; estudiantes universitarios; principiantes; modalidad virtual

\section{Abstract}

The purpose of the research is to characterize the learning styles of beginning university students with virtual modality, describing the preponderance of each of the styles proposed by Felder and Silverman in 1988. In methodological terms, it is a mixed study of exploratory-descriptive character in which 5951 students participated, attached to academic programs oriented from virtuality. The main results highlight the predominance in the students of learning styles oriented to the active, reflexive and visual. The conclusion is that it is pertinent to identify actions that meet the needs of students in such a way that they feel motivated and strengthen their sense of institutional belonging, in such a way that they appropriate the didactics of virtual mediation and improve their academic performance.

Keywords: Learning styles; university students; beginners; virtual modality 


\section{INTRODUCCIÓN}

Dentro del ambiente académico ha sido un constante reto crear modelos educativos pertinentes y nuevas apuestas formativas relacionadas con los procesos de aprendizaje. En lo que tiene que ver con la educación a distancia en ambientes virtuales de aprendizaje, específicamente en las últimas décadas se ha prestado especial interés a la innovación permanente y a la forma cómo se comprende la dinámica del aprendizaje a fin de enriquecer los procesos educativos teniendo en cuenta no sólo los aspectos institucionales, sino también los aspectos individuales de los estudiantes que aprenden y de los facilitadores del aprendizaje.

En este artículo se caracterizan los estilos de aprendizaje de los estudiantes de primera matrícula de la Universidad Nacional Abierta y a Distancia -UNAD- de Colombia con Modalidad virtual, tomando como referentes los constructos teóricos sobre estilos de aprendizaje de Felder y Silverman (1988), quienes comprobaron que los estudiantes aprenden de diferentes maneras: por el oír y ver; reflexionando y actuando; razonando de manera lógica o intuitiva; memorizando y visualizando y estableciendo analogías; o de manera constante, en pequeños trozos o piezas de gran tamaño; y defienden que los estilos de enseñanza varían, como la preferencia de un educador para utilizar estrategias que faciliten la adquisición de conocimientos y habilidades para aplicar dichos conocimientos (Felder y Spurlin, 2005).

Se describen los criterios metodológicos para caracterizar los estilos de aprendizaje de los estudiantes de primera matrícula durante tres periodos del año 2017, y se identifican las tendencias predominantes en los estilos de aprendizaje de estos estu- diantes, con el propósito de recoger elementos que fortaleciendo las prácticas pedagógicas y favoreciendo el diseño de cursos y materiales, redunden en la maximización de los procesos educativos en ambientes virtuales.

Se hace necesario reconocer cuáles son los estilos de aprendizaje de los estudiantes y desde allí poder rediseñar la forma en que se presentan los contenidos e implementar acciones de mejora en los diseños instruccionales y en las propuestas de desarrollo de las actividades enriqueciéndose con el uso de multiformatos, que sean consecuentes con las formas y tendencias de aprendizaje de los estudiantes de primera matrícula de la universidad virtual donde se realizó el estudio.

Describir e identificar las tendencias los estilos de aprendizaje de los estudiantes de primera matrícula en la modalidad virtual es de gran relevancia en los procesos de formación universitaria dado que el conocimiento de estos estilos contribuye al diseño instruccional respondiendo a las necesidades de aprendizaje de los estudiantes, para que partiendo del reconocimiento de la diversidad se puedan enriquecer las prácticas pedagógicas, la construcción de actividades y el diseño contenidos de los cursos académicos.

\section{Referentes Conceptuales}

En el ámbito académico específicamente cuando se habla de educación a distancia en ambientes virtuales de aprendizaje, la innovación permanente que rompe con los viejos conceptos pedagógicos ha posibilitado avances en la forma como se comprende, pero también en la manera de superar los desafíos que enfrenta el proceso educativo en esta modalidad. El quehacer de los estudiantes, especialmente en modalidad vir- 
tual, implica unas demandas académicas, sociales y tecnológicas que implican la actualización constante en información científica y la evaluación permanente, además, de largar horas de estudio (Ferrel, Thomas, Solano, Redondo \& Ferrel, 2014). Los factores que repercuten en el proceso de aprendizaje como, métodos de enseñanza, estrategias de aprendizaje, conocimientos previos de los estudiantes, procesos de evaluación que aplica el docente, entre otros, pueden facilitar o inhibir el proceso que aún se encuentra en permanente estudio, en favor de mejorar el desempeños tanto de los docentes como de los estudiantes.

Para definir el concepto de estilo de aprendizaje es preciso delimitar las áreas que abarca y las aplicaciones que describe, aquello que es común a estas diversas formas de procesar la información cuando se aprende (Witkin, 1985). Los estilos de aprendizaje no solamente afectan nuestra manera de aprender, sino que fundamentalmente intervienen en nuestra manera de pensar, ya que el pensamiento es el punto de partida del aprendizaje (Pearson, 2007).

No existe una única definición de estilos de aprendizaje. Para Keefe (1988) los estilos de aprendizaje son rasgos cognitivos, afectivos y fisiológicos, que sirven como indicadores relativamente estables de cómo los alumnos perciben interacciones y responden a sus ambientes de aprendizaje (Dunn, Dunn \& Price, 1985) expresan que el estilo de aprendizaje es la manera en la que un aprendiz comienza a concentrarse en una información nueva y difícil, la analiza, la incorpora, la trata y la retiene (Hunt citado por Chevrier, Fortin, Théberge \& Le Blanc, 2000) establece que un estilo de aprendizaje es aquel que describe la forma como un aprendiz adquiere el conocimiento en términos de condiciones educativas que favorecen su aprendizaje de manera eficiente. Mientras Willing (1988) y Wenden (1991) explican cómo la noción de estilo de aprendizaje se superpone a la de estilo cognitivo, pero es más comprensiva porque incluye comportamientos cognitivos $\mathrm{y}$ afectivos que determinan la forma de percibir, interactuar y responder al contexto de aprendizaje.

Cada persona aprende de manera diferencial es decir aplicando diferentes estrategias a distinta velocidad con diversos niveles de motivación y de forma dinámica. Es por eso que autores como Revilla (1998) establecen que los estilos de aprendizaje son formas relativamente estables que no se expresan nunca en categorías cerradas y que pueden ser cambiantes de forma situacional, pueden mejorar y pueden potenciarse cuando se utiliza el propio estilo de aprendizaje para aproximarse al conocimiento. Si bien los estilos de aprendizaje corresponden más a modelos teóricos siempre llevan implícito un comportamiento que predetermina la conducta de aprendizaje y el procesamiento de la información en los individuos marcando una tendencia.

Así los diversos estilos de aprendizaje actuarían como instrumentos heurísticos que permiten analizar el comportamiento de un individuo al momento de aprender. Algunos autores como Woolfolk (1996) sugieren hablar de preferencias de estilos de aprendizaje para explicar las formas predilectas para estudiar y adquirir conocimientos y habilidades ofreciendo un marco conceptual que puede llegar a explicar el comportamiento a la hora de aprender. Existen varios modelos de estilos de aprendizaje (Chevrier et al, 2000; Garza y Leventhal, 2000; Jensen, 1994; Chavero, 1999; Cazau, 2001) que pueden llegar a convertirse en clasificaciones diversas. 
Entre esas clasificaciones es bastante reconocido el modelos "Orion" desarrollado por Curry (1987), el cual señala las preferencias al momento de aprender y las coloca en categorías como las preferencias ambientales (sonido, luz, temperatura, distribución de espacios), preferencias emocionales (motivación, voluntad, responsabilidad), preferencias de interacción social (estudio individual e independiente, colaborativo en parejas, grupal, participativo o no participativo), preferencias fisiológicas (percepción, tiempo, movilidad) y preferencias psicológicas (basadas en modos analíticos y hemisferios cerebrales, cortical-límbico, concreto-abstracto, activo-pensativo, visual-verbal, inductivo-deductivo, secuencial-global) y las dimensiones de personalidad (extrovertidos-introvertidos, sensoriales-intuitivos y racionales-emotivos).

Por su parte Dunn, Dunn \& Price (1985) en su inventario teoría de estilos de aprendizaje pudieron establecer y medir veintiún (21) elementos que configuran lo que ellos llamaron gustos personales en la forma de aprender, estableciendo que cada persona aprende a su modo y por factores condicionantes (fisiológicos, psicológicos, sociológicos, emocionales, comportamentales). En esta misma línea de modelos construidos con escalas de medición, se encuentra también el modelo de Keefe y Monje (citado en Roset, 2003) Learning Style Profile (LSP), que evalúa los estilos cognitivos en estudiantes de secundaria y los clasifica en 23 variables agrupadas en tres factores que influyen en el aprendizaje: habilidades cognoscitivas (analítico, espacial, discriminatoria, tratamiento secuencial, memorística), percepción de la información (visual, auditiva y verbal), y preferencias para el estudio y aprendizaje (perseverancia, deseo, preferencia para la manipulación, preferencia para trabajar por las mañanas o las tardes, preferencias teniendo en cuenta el agrupamiento, y preferencias relativas a la movilidad, sonido, iluminación y temperatura).

Otro modelo usado en la clasificación de estilos de aprendizajes es el Inventario de estilos de aprendizaje de Canfield (Canfield's Learning Styles Inventory) que caracteriza la tipología de los estudiantes con base en cuatro categorías: condiciones para aprender (organización del curso, competencia, relación con el profesor), cómo debe ser detallada la información (independencia, autoridad, áreas de interés), grado o nivel de conocimiento en relación con los otros (superior, nivel bueno, promedio o satisfactorio, por debajo del promedio o insuficiente). Grasha \& Riechman (1975) teniendo en cuenta el contexto del aprendizaje en grupos, desarrollan un modelo basado en las relaciones interpersonales; el instrumento que ellos elaboran, el Grasha-Reichmann Learning Style Scales (GRSLSS), que supone la existencia de tres dimensiones bipolares: autónomos/dependientes, colaborativos/competitivos y participativos/ no participativos.

Basándose en los modelos de Sperry y de McLean, Herrmann (citado en Roset, 2003) se elaboró un modelo, el «Herrmann Brain Dominance Instrument (HBDI)», el cual percibe el cerebro compuesto por cuatro cuadrantes, que resultan del entrecruzamiento del hemisferio izquierdo y el derecho del modelo Sperry, y de los cerebros límbico y cortical del modelo McLean.

Otro Modelo englobado en esta categoría es el modelo de Kolb, denominado «Experimental Learning» (Kolb, 1984). Este supone que para aprender algo se debe trabajar o procesar la información recibida, partiendo de una experiencia directa y concreta, y de una experiencia abstracta, la cual se tiene cuando se lee o se escucha acerca un tema. 
Las experiencias que se tienen (concretas o abstractas) se traducen en conocimientos cuando se elaboran de alguna de estas dos formas: reflexionando y pensando sobre ellas o experimentando de forma activa con la información recibida. En este modelo según el autor, cada persona se especializa dependiendo de la forma como desee aprender y se convierte en Divergentes (se basa en experiencias concretas y la observación reflexiva), Convergente (utiliza la conceptualización abstracta y la experimentación activa), Asimilador (usa la conceptualización abstracta y la observación reflexiva, planeando y fijándose metas) y Acomodador (basado en la experiencia concreta y la experimentación activa, son adaptables, intuitivos y aprenden por ensayo y error).

Honey y Mumford, con base en la teoría de Kolb establecieron cuatro estilos de aprendizaje (Alonso, Gallego y Honey, 1994): activos, reflexivos, teóricos y pragmáticos. Por su parte Mc Carthy (1987), basándose en la idea del aprendizaje experiencial de Kolb (1984), atribuye diferencias en los estilos de aprendizaje de las personas, según cómo perciban o procesen información: describiendo cuatro grandes estilos de aprendizaje: el imaginativo (percibe información concreta, reflexiona y la integra con sus experiencias, asignándole un significado y valor), el analítico (percibe la información en forma abstracta y mediante la observación reflexiva, la mayor fortaleza de este aprendiz está en el razonamiento inductivo y la habilidad para crear modelos teóricos), el del sentido común (confía en la conceptualización y experimentación activa, integra la teoría con la práctica, la solución de problemas y la toma de decisiones convergen muy bien en la aplicación práctica de las ideas) y el dinámico (tiene la fortaleza opuesta al asimilador, enfatiza la experiencia concreta y la experimentación activa y percibe la información en forma concreta, adapta, es intuitivo, trabaja sobre el ensayo-error).

Existen también teorías basadas en los postulados de la programación neurolingüística como la de Bandler y Grinder citados por Pérez (2001), que integran los sistemas de representación visual, representación auditiva, representación kinestésica. Otras de las teorías relevantes es la de Pearson (2007) donde se define el estilo de aprendizaje como un conjunto de características biológicas y madurativamente impuestas asociado la efectividad de un método de enseñanza. Esta autora expresa para cada persona un estilo determinado por características biológicas e innatas, pero también por la estimulación recibida y es por ello que se potenciarán si se facilita y permite el procesamiento de la información, así como si se reconocen factores propios y dificultades específicas en cada uno de los actores del proceso de aprendizaje.

Otro de los modelos es el de estilos de aprendizaje de Felder y Silverman (1988), cuya clasificación la hace a partir de cinco dimensiones, las cuales están relacionadas con las respuestas obtenidas en una serie de preguntas. El estilo de aprendizaje de un estudiante vendrá dado por la combinación de las respuestas obtenidas en las dimensiones establecidas por estos autores. Durán y Costaguta (2007) recogen y describen las principales características del modelo de estilos de aprendizaje de Felder y Silverman (1988) y estas características se contrastan con las descritas por Felder y Brent (1996) que de forma condensada se pueden describir así:

- Aprendizaje visual: Los estudiantes visuales recuerdan mejor lo que ven, es decir prefieren representaciones y apoyos gráficos como las figuras, los diagramas, los cuadros, las líneas de tiempo, las películas, o las demostraciones. 
- Aprendizaje verbal: Esta categoría inicialmente se conceptualizó como estilo auditivo, pero fue reemplazada por los contenidos verbales que incluyendo los auditivos marcan la inclinación a recordar más lo que se escucha y mucho más lo que se dice. Los estudiantes con este estilo aprenden a partir de la discusión y prefieren las explicaciones verbales a las demostraciones visuales. Aprenden efectivamente cuando les explican otros y prefieren las explicaciones escritas o habladas.

El proceso mental complejo por el cual la información percibida es convertida en conocimiento puede ser convenientemente agrupado en las siguientes dos categorías que hacen parte del modelo, la experimentación activa (aprendizaje activo) y la observación reflexiva (estilo reflexivo).

- Aprendizaje activo: este estilo facilita la recepción de información y la transformación de la misma con la experimentación y el ensayo. Implica hacer algo en el mundo externo con la información ya sea discutirla, explicarla o chequearla de alguna manera. Un estudiante activo es el que se siente más cómodo con la experimentación activa que con la observación reflexiva. Los estudiantes activos no aprenden mucho en situaciones en las que ellos deben estar pasivos, tales como lecturas; trabajan bien en grupos, tienden a ser experimentalistas y aprenden manipulando las cosas.

- Aprendizaje Reflexivo: Esta otra forma de recibir información y convertirla en conocimiento implica examinar y manipular la información introspectivamente. Un estudiante reflexivo se siente más cómodo con la observación reflexiva que con la experimentación activa. Los estudiantes reflexivos no aprenden bien en situaciones que no les proporcionan la oportunidad de pensar sobre la información que se les presenta; trabajan mejor solos o a lo sumo con una persona más. Tienden a ser teóricos, reflexivos, prestando más atención a pensar sobre las cosas y a trabajar solos.

Otros estilos de aprendizaje están conformada por el aprendizaje de progresión (secuencial) y el aprendizaje holístico (global). Al respecto se destaca lo siguiente:

- Aprendizaje Secuencial: El aprendizaje secuencial es el aplicado en la mayoría de la educación formal, e implica la presentación de material en un orden de progresión lógica, con el avance del aprendizaje regido por el tiempo y el calendario. Los estudiantes con este estilo siguen la secuencia de las indicaciones y construyen de forma escalonada y progresiva. En este estilo cuando los estudiantes han abarcado un tema y son evaluados positivamente pueden pasar al siguiente nivel, permitiendo un aprendizaje secuencial, paulatino y de forma ordenada y acumulativa.

- Aprendizaje Global: El aprendizaje global es aquel que no se rige por el tiempo ni el calendario. Los estudiantes con este estilo pueden pasar días o semanas ocupados en resolver un simple problema o demostrando una comprensión rudimentaria articulando varias teorías o perspectivas del objeto de estudio hasta que logran una rápida comprensión o la resolución del mismo o de la tarea. Este estilo privilegia en los estudiantes la comprensión del todo, la aproximación global y el aprendizaje de forma sincrética. 
Por último se tiene lo sensorial-intuitivo; implica dos categorías que involucran la percepción en un sentido más amplio:

- Aprendizaje sensorial: Implica observar y recopilar datos a través de los sentidos, aunque este estilo de aprendizaje aparece muy relacionado con el intuitivo, sin embargo, los estudiantes suelen privilegiar uno de los dos estilos de la bina sensorial- intuitiva. Los estudiantes sensoriales son pacientes, hacen las cosas con detalle, no les gustan las complicaciones. Los sensores son buenos para memorizar hechos, y tienden a ser cuidadosos en la realización de su trabajo.

- Aprendizaje intuitivo: Privilegia la intuición que implica la percepción indirecta a través del inconsciente: la especulación, la imaginación y corazonadas. Aunque los estudiantes usarán ambas de estas facultades, la mayoría se prefiere el uso de uno u otro. Los estudiantes intuitivos son innovadores, se inclinan por el descubrimiento y la abstracción. Prefieren los principios, las teorías y la innovación, pero no les gusta la repetición. Los intuitivos son buenos en captar nuevos conceptos, y tienden a completar tareas rápidamente.

Estos estilos de aprendizaje pueden combinarse conformando otras categorías y nuevos estilos, subsanando la crítica inicial de que no existe una única forma de acceder, procesar y reconstruir la información generando aprendizajes. Las categorías emergentes que resultan de las combinaciones de los estilos descritos anteriormente abren la posibilidad de expresar de mejor forma las particularidades de cómo se aprende. Retomando el enfoque de Felder y Silverman (1988), numerosos estudios latinoamericanos, entre los que destacan los aportes mexicanos de Aragón y Jiménez, 2009; García, Santizo y Alonso, 2009; Rodríguez, Fajado, Figueroa y González, 2006 y Valadez, 2009 al enunciar como alternativas de integración de los estilos la posibilidad de caracterizar los estilos emergentes.

Los integran de la siguiente manera: a) Estilo intuitivo-reflexivo: Relaciona información concreta con abstracciones y conceptualizaciones formales. b) Estilo visual-sensitivo: Combina el uso de dibujos, gráficos, tablas, fotos y demostraciones, con exposiciones magistrales del estilo verbal. c) Estilo activo-reflexivo: Alterna modalidades de discusión grupal y de reflexión individual. d) Estilo secuencial-sensitivo: Entrelaza explicaciones que sigan un orden sucesivo y constante que conduzca al entendimiento de lo particular a lo general, forjando métodos preestablecidos de pensamiento. e) Estilo global-intuitivo: Con exposiciones que parten de las relaciones y requieran grandes saltos en la comprensión. f) Estilo reflexivo-global: Apela a la formulación de distintos tipos de interrogantes que demanden respuestas creativas, concretas, prácticas, orientadas hacia los hechos. g) Estilo sensitivo-intuitivo: Combina los procedimientos sensitivos o conceptuales, innovadores, orientados hacia la asimilación de las teorías de forma intuitiva. h) Estilo inductivo-deductivo: Prefiere la información que deviene desde lo específico hacia lo general o prefieren la información que deviene desde lo general hacia lo específico.

A continuación, se muestra de forma gráfica las características de aprendizaje de los estudiantes, según las dimensiones del modelo (Figura 1). 


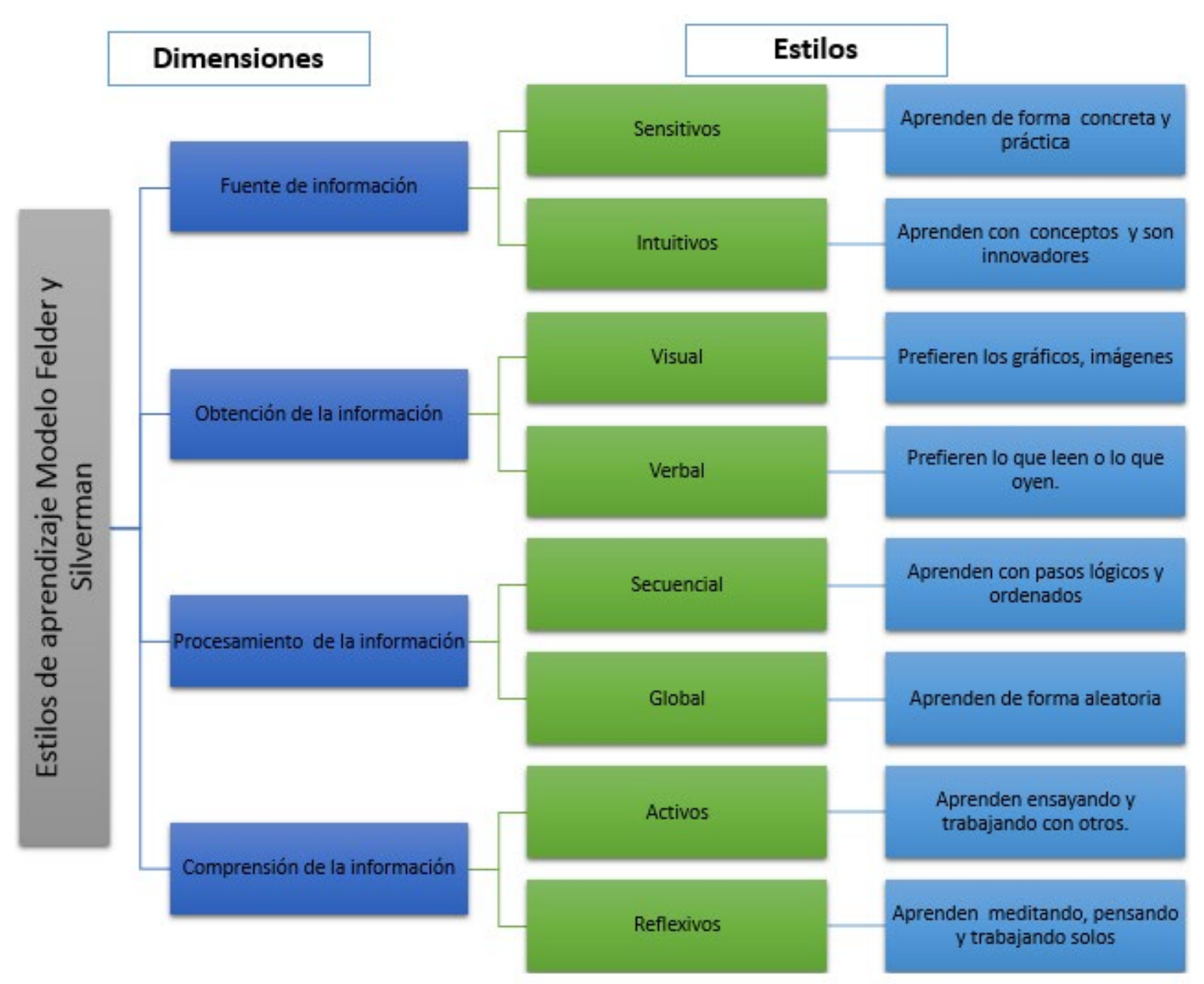

Figura 1. Estilos de aprendizaje - Modelo Felder y Silverman.

Fuente: elaboración propia.

En este modelo no hay estilos correctos de aprendizaje; más bien, se entiende como un sistema de preferencias en el cual participan los estudiantes de manera individual. Este modelo presenta ciertas analogías con otros tres modelos de estilos de aprendizaje: el modelo de Kolb, un modelo basado en el indicador de Myers-Briggs y una aplicación del instrumento de Herrmann basado en la especialización de los hemisferios del cerebro (Felder y Brent, 1996). Los planteamientos de Felder y Silverman (1988) confirman que los estudiantes difieren en la manera de acceder al conocimiento en términos de intereses y estilos, en el sentido de poseer puertas de entrada diferentes para iniciar el proceso del conocimiento (Litwin, 1997), pero también invitan a pensar que puede ser de gran utilidad para los docentes conocer las formas particulares de acceder a la información.
En este sentido, Felder y Brent (citado por Aragón y Jiménez, 2009) reseñan algunas de las estrategias utilizadas para que la información presentada en el aula tenga en cuenta los diferentes estilos de aprendizaje de los aprendices:

Para los estilos sensitivo, inductivo y global, el docente antes de presentar el material teórico, debe describir problemas o fenómenos que guarden relación con dicho material, incluso permitirles tratar de resolver cuestiones relacionadas al tema de tal forma que logren percibir la necesidad de nuevas herramientas.

Para el estilo sensitivo, intuitivo, visual y verbal el docente debe balancear la información concreta, con la conceptual. Debe intensificar el uso de diagramas, esquemas, gráficos y demostraciones, junto con exposiciones orales y lecturas. 
Para el estilo sensitivo deberá privilegiar la ilustración de conceptos abstractos con uno o varios ejemplos, o utilizar analogías físicas y demostraciones para ilustrar los contenidos.

Para los estilos reflexivo y activo, debe dar tiempo a los estudiantes en la clase para reflexionar acerca del material presentado y para la participación con actividades tales como relevar los puntos más importantes de una lectura o por la resolución de problemas en pequeños grupos, así como encargar que algunas tareas extra clase sean resueltas en forma cooperativa.

Para los estilos secuencial y global, tendrá entonces que mostrar el flujo lógico de los temas a abordar y también las conexiones de estos con otros temas similares o contingentes, o con otros de la misma disciplina, de otras disciplinas y/o de la vida diaria.

Si bien son diversas las clasificaciones de los estilos de aprendizaje, en lo que todas las teorías confluyen es en que cada persona tiene sus estrategias particulares y sus propios métodos para adquirir los conocimientos y aprender. Aunque el uso de uno $\mathrm{u}$ otro método o estrategia depende muchas veces del contexto, se puede afirmar que existen tendencias globales para definir un estilo propio de aprendizaje y estos estilos de aprendizaje son útiles no solamente desde la perspectiva del estudiante sino desde la perspectiva del docente, ya que ambos pueden presentar y recibir la información de manera más efectiva y según las características particulares de quien presenta y de quien recibe.

Lo cierto es que los estilos de aprendizaje también afectan la forma cómo se procesa la información y sobre todo el que los seres humanos reconozcan la propia forma de aprender y las preferencias al momento de recibir e interiorizar la información, son verdaderamente importantes para gestio- nar procesos de aprendizaje; pero lo es más aún el que los docentes reconozcan la forma de pensar, la forma cómo los estudiantes se acercan al aprendizaje y las preferencias de cada uno, ese es el primer paso para ser más efectivos en su trabajo con diversidad de estudiantes (Pearson, 2007). Desde esta perspectiva no solo es importante reconocer el estilo de aprendizaje del estudiante, sino el estilo de pensamiento ya que este incidirá directamente en su estilo de enseñanza.

\section{MetodoloGía}

Se trabaja bajo un enfoque mixto; representa una forma práctica de ahondar en el conocimiento científico, puesto que en sí integra un componente cualitativo y otro cuantitativo; según plantean Johnson \& Onwuegbuzie (2004) la investigación de métodos mixtos (investigación mixta como sinónimo) es el complemento natural de la investigación tradicional cualitativa y la cuantitativa; lo que Senior, Colina, Marín y Perozo (2012) denominan enfoque multimétodo.

Esta investigación tiene el carácter de exploratoria, puesto que no existen estudios realizados bajo esta perspectiva y enfoque sobre los estilos de aprendizaje en la población específica de estudiantes de la modalidad virtual en Colombia. Acorde a los objetivos establecidos y la naturaleza del ejercicio, el diseño se orienta a la investigación no experimental de carácter transeccional o transversal (Hernández, Fernández y Baptista, 2010). La población de estudio estuvo conformada por 8.147 estudiantes de primera matricula y la muestra representativa de tipo no probabilístico fue de 5.951 estudiantes divididos en tres grupos por periodos de matrícula. A continuación, se describen las características sociodemográficas de los participantes. 
El 51.8\% de los estudiantes que diligenciaron la encuesta pertenecen al género femenino y el $48.2 \%$ al masculino. El $63.66 \%$ de los estudiantes estaban solteros, el $17.06 \%$ casados, el $16.24 \%$ en unión libre, el $1.56 \%$ separados. El 1.37\% (130 estudiantes) eran madres solteras cabeza de hogar, el $0.08 \%$ (8 estudiantes) viudos y el $0.03 \%$ (2 estudiantes) religiosos. El 30.9\% de los participantes pertenece era de estrato 1 , el $42.61 \%$ pertenecía al estrato 2 , el $22.83 \%$ al estrato 3 , el $2.97 \%$ al estrato $4,0.56 \%$ pertenecía al estrato 5 y el $0.13 \%$ al estrato 6 .

El $31 \%$ de los estudiantes encuestados tenía entre 21 y 25 años, el $22.3 \%$, corresponde a estudiantes entre los 15 a los 20 años, el $22,2 \%$ a estudiantes entre los 26 y los 30 años, el $12.8 \%$ corresponde a estudiantes de 31 a 35 años y el 11.7\% corresponde a estudiantes de 36 años en adelante. El $60.11 \%$ de los encuestados estaban empleados, el $31.16 \%$ se encontraban desempleados, el $8.07 \%$ son independientes y el $0.66 \%$ son empresarios.

Las técnicas empleadas para la recolección de la información son la encuesta y la observación. Para el caso de esta investigación se utiliza la encuesta descriptiva, que permite recoger información de modo estandarizado usando el cuestionario de Felder y Silverman (1988), maneja cuatro dimensiones dicotómicas, y está conformado por 44 preguntas; este instrumento permite establecer los índices de estilos de aprendizaje y describir sus tendencias en cada uno de los sujetos.

Para el caso de esta investigación se utiliza también la observación no sistemática y no participante (Hernández, Fernández y Baptista, 2010), que tiene como referente el comportamiento de los estudiantes en el contexto de aprendizaje dentro del curso y se fundamenta en la sensibilidad para descubrir elementos y factores importantes complementarios a los hallazgos de la encuesta.
Respecto del procedimiento de aplicación del instrumento, los participantes reciben en el aula del campus virtual instrucciones de responder las preguntas de este cuestionario, indicando el propósito de la aplicación. De igual forma se giran instrucciones específicas para responder la encuesta, en relación con una adecuada conexión a internet, la disposición del tiempo para contestar las preguntas (2 horas máximo), se explica la estructura de la prueba, el tipo de preguntas usado y se hace énfasis en aspectos como que no hay respuestas correctas o incorrectas y en la necesidad de responder todas las preguntas.

Para salvaguardar los aspectos éticos en el ejercicio de recolección de la información se solicita consentimiento a cada uno de los participantes y la manifestación de su aceptación antes de empezar a responder. Además, no se solicita el nombre del participante ni datos específicos distintos a la información demográfica. Las respuestas son alojadas en una base de datos creada para generar los resultados de la aplicación.

\section{Resultados}

Al consolidar la información de la encuesta de estilos de aprendizaje de los tres periodos académicos correspondientes al 2017, se evidencia una participación total de 5.951 estudiantes que la respondieron (Tabla 1 ).

TABla 1.

Número de estudiantes que realizaron la encuesta por periodo

\begin{tabular}{lc}
\hline $\begin{array}{c}\text { Periodo académico del 2017 y } \\
\text { semanas de duración }\end{array}$ & $\begin{array}{c}\text { Número de } \\
\text { estudiantes }\end{array}$ \\
\hline $\begin{array}{l}\text { Periodo } 1 \\
\text { (duración } 16 \text { semanas) }\end{array}$ & 3244 \\
$\begin{array}{l}\text { Periodo } 2 \\
\text { (duración } 16 \text { semanas) }\end{array}$ & 1706 \\
$\begin{array}{l}\text { Periodo } 3 \\
\text { (duración } 8 \text { semanas) } \\
\text { Total de la Muestra }\end{array}$ & 1001 \\
\hline
\end{tabular}

Fuente: elaboración propia. 
Para el análisis se tiene en cuenta el consolidado de los tres periodos académicos del 2017, teniendo en cuenta las 4 dimensiones de estilos aprendizaje: activo- reflexivo, sensorial-intuitivo, visual-verbal, secuencial-global y en cada una de estas dimensiones, las respuestas a las preguntas se organizaron en rangos A y B como lo referencian los autores, por ejemplo la dimensión Activo (A) Reflexivo (B) y los resultados se tabulan teniendo en cuenta las preguntas que definen el estilo de aprendizaje del estudiante. Los puntajes son analizados de acuerdo a los rangos y plano cartesiano para las 4 dimensiones propuestos por Felder y Spurlin (2005) como se muestra en la Tabla 2.

TABLA 2.

Dimensiones evaluadas en la encuesta de estilos de aprendizaje

\begin{tabular}{lcc}
\hline \multicolumn{1}{c}{ Dimensiones } & Rangos A & Rangos B \\
\hline $\begin{array}{l}\text { Activo - } \\
\text { reflexivo }\end{array}$ & $11-9-7-5-3-1$ & $1-3-5-7-9-11$ \\
$\begin{array}{l}\text { Sensorial - } \\
\text { intuitivo }\end{array}$ & $11-9-7-5-3-1$ & $1-3-5-7-9-11$ \\
$\begin{array}{l}\text { Visual- verbal } \\
\begin{array}{l}\text { Secuencial - } \\
\text { global }\end{array}\end{array}$ & $11-9-7-5-3-1$ & $1-3-5-7-9-11$ \\
\hline
\end{tabular}

Fuente: elaboración propia.

La información resultante del análisis de la encuesta realizada muestra las tendencias de los estilos de aprendizaje en estu- diantes de los tres periodos del 2017; estas tendencias son contrastadas con el análisis estadístico derivado de la información obtenida. A continuación, aparecen las puntuaciones de la media para cada una de las categorías de estos periodos (Tabla 3).

En la Tabla 3 se puede corroborar como las puntuaciones medias en cada una de las categorías son muy similares a las tendencias obtenidas con el análisis del instrumento que se evidencia en la Tabla 2. De forma descriptiva se encuentra nuevamente establecido en la puntuación estadística que en el periodo 1 el estilo visual es el que tiene la tendencia más alta y en consecuencia la puntuación media es la mayor lo que indica que es la tendencia de estilo de aprendizaje según orden de importancia. Seguido por el estilo activo que tiene una puntuación media de 1957,81818. La puntuación media última le corresponde al estilo sensorial que obtiene una media de 1947,36364. Se observa también que la puntuación mínima la conserva el estilo verbal, seguido por el estilo reflexivo y en su orden por el intuitivo y el global. En el periodo 1, la tendencia de los 3.244 estudiantes de acuerdo a las respuestas registradas por parte de ellos determina que son más activos que reflexivos, más sensitivos que intuitivos y más secuenciales que globales, esto manifiesta que los estudiantes requieren de estrategias de aprendizaje que se articulen a estas formas de aprender.

TABla 3.

Puntuaciones medias de las tendencias por periodos

\begin{tabular}{ccccccccccc}
\hline Periodos & Dimensiones & Activo & Reflexivo & Sensorial & Intuitivo & Visual & Verbal & Secuencial & Global \\
& Tendencias & A & B & A & B & A & B & A & B \\
1 & Media & 1957,81818 & 1286,18182 & 1847,36364 & 1296,09091 & 2113,36364 & 1130,63636 & 1934,18182 & 1309,81818 \\
2 & Media & 1039,7272 & 666,2727 & 1013,3636 & 692,6363 & 1045,6363 & 660,3636 & 1011,4545 & 694,5454 \\
3 & Media & 615,7272 & 385,272 & 663,6363 & 436,3636 & 654,4545 & 346,5454 & 594,6363 & 406,363 \\
\hline
\end{tabular}

Fuente: elaboración propia. 
En la Tabla 3, aparecen también las puntuaciones de la media estadística correspondientes al período 2 , información cuantitativa que corrobora los resultados obtenidos del análisis directo del instrumento a partir de la obtención de las puntuaciones medias de las respuestas de los estudiantes. Se observa para este periodo una tendencia similar, privilegiando el estilo visual con una puntuación media de 1045,6363 seguido por el estilo activo, que serían los dos estilos predominantes en los estudiantes según las puntuaciones medias. En ese mismo orden de ideas el estilo verbal con una puntuación media de 660,3636 y el reflexivo con puntuación de 666,2727 son los estilos de aprendizaje menos utilizados por los estudiantes. En este periodo 2, la tendencia de los 1.706 estudiantes que respondieron la encuesta, presenta un equilibrio entre lo sensorial y lo intuitivo y lo secuencial y global. Estos estudiantes tienden a aprender y comprender el mundo a través de formas diversas, es decir desde lo sensorial observan y recopilan datos a través de los sentidos, y desde lo intuitivo aprenden y se acercan a la nueva información desde la especulación, la imaginación y simples corazonadas.

Finalmente, en el período 3 de la Tabla 3, el comportamiento es igual al de los periodos anteriores privilegiando en la tendencia el estilo visual, seguido por el activo y el sensorial. Para el periodo 3, la tendencia de los 1.101 estudiantes que respondieron la encuesta, se caracteriza por tener un equilibrio entre lo activo- reflexivo, lo sensorial y lo intuitivo, lo visual y auditivo y lo secuencial y global, lo que evidencia que son estudiantes muy equilibrados en su proceso de aprendizaje y se caracterizan por observar y recolectar la información a través de los sentidos como el gusto, el tacto, el olfato y la intuición que implica que usen la percepción indirecta a través del inconsciente.
De acuerdo a lo anterior se puede decir que, en los estudiantes encuestados, resaltan para cada dimensión respuestas en el rango de activos, sensoriales, visuales y en igual medida la dimensión secuencial-global. Estos estilos predominantes identificados mediante las encuestas permiten establecer además las necesidades de aprendizaje de los estudiantes y las formas, adaptaciones y actividades que desde la acción docente se deben incorporar para lograr que los estudiantes de primera matrícula se sientan cómodos, acogidos, identificados con las actividades, los temas, las formas de presentación de los contenidos, los recursos entre otros y así logren el éxito académico.

De otro lado al relacionar las tendencias en las respuestas arrojadas por la encuesta, con las observaciones y reflexiones manifestadas por los estudiantes en las webconferencias del curso y los encuentros b-learning, estrategias de interacción sincrónica y asincrónica entre estudiantes y tutores de carácter pedagógico-didáctico que buscan fortalecer los aprendizajes significativo, colaborativo y autónomo en los estudiantes; se encuentra una coherencia importante en lo expresado por éstos al evaluar dichas estrategias, afirmando que el aporte de las webconferencia respecto al contenido y desarrollo de las actividades del curso fue excelente para un $79 \%$ y buena $21 \%$ (859 encuestados). Del mismo modo al analizar las respuestas de la evaluación que los estudiantes realizan de los encuentros b-learning, se descubre que el incorporar en los cursos actividades de orden lúdico-práctico, desde la gamificación, el trabajo colaborativo, la implementación de recursos en multiformatos y la explicación paso a paso de las acciones a realizar; se facilita a los estudiantes aproximarse al conocimiento de temas y contenidos de una forma más apropiada según sus tendencias en los estilos de aprendizaje. 


\section{Discusión DE RESULTADOS}

Una vez recolectada la información con la aplicación del cuestionario de estilos de aprendizaje de Felder y Silverman (1988) se encuentra como tendencia principal de los estudiantes el aprendizaje visual, el aprendizaje activo, el aprendizaje sensorial y el secuencial invitando a preferir situaciones de la vida real y aprender con los hechos de forma visual y práctica. Se encuentran y describen también otras tendencias de aprendizaje que de forma significativa caracterizan el aprendizaje de los estudiantes de primera matrícula.

Al relacionar las tendencias arrojadas por la aplicación del cuestionario nos aproximamos a la comprensión de la forma como aprenden los estudiantes en la modalidad virtual y a partir de allí extraer aplicaciones prácticas relacionadas con el diseño de materiales y contenidos, la selección de las temáticas y las acciones pedagógicas, y también se pueden identificar elementos que desde la perspectiva del estudiante al reconocer su estilo de aprendizaje autorregula su proceso de formación con ejercicios metacognitivos que fortalezcan la experiencia de formación académica.

Así también, se advierte la relación entre estilos y estrategias de aprendizaje, lo cual confirma el planteamiento de Aguirre, Cancino y Loaiza (2009), quienes aducen en cuanto a los estilos que "los alumnos conforme avanzan en su proceso de aprendizaje descubren mejores formas o modos de aprender, por lo tanto, van a variar su estilo, además dependerá de las circunstancias, contextos y tiempos de aprendizaje que tengan que enfrentar" (p. 12). Con respecto a las estrategias, los resultantes se contrastan con los planteamientos de Díaz-Barriga, Castañeda \& Lule y Hernández (citados por Díaz-Barriga y Hernández, 2003), al expre- sar que "una estrategia de aprendizaje es un procedimiento (conjunto de pasos o habilidades) que un alumno adquiere y emplea de forma intencional como instrumento flexible para aprender significativamente y solucionar problemas y demandas académicas" (p. 12). Adicionalmente se confirma el planteamiento de Camarero, del Buey y Herrero (2000) en relación con que los estilos y estrategias específicas de aprendizaje entre los estudiantes que ven un curso en línea lo hacen más accediendo a recursos visuales y en función de la búsqueda de relaciones entre los contenidos; esto es buscan mas estrategias de adquisición de la información basadas en lo visual y usan como técnicas de aprendizaje las que se basan en el repaso en voz alta y en el establecimiento de reflexiones empleando el estilo activo y pragmático de aprendizaje.

\section{Conclusiones}

Los estudiantes de primera matrícula participantes en el estudio presentan una tendencia en su estilo de aprendizaje referido en su mayoría a lo activo, reflexivo y visual, esto se constituye en una fortaleza dado el modelo pedagógico de la universidad donde se realizó el estudio (UNAD) y el aprendizaje a través del campus virtual y de la información que en mayor medida está presentada de forma visual en los cursos de primera matrícula para la comprensión de los contenidos y temáticas por parte de los estudiantes. Desde la perspectiva del ejercicio docente y dado que los estudiantes expresan en puntuaciones significativas una tendencia al desarrollo secuencial planear actividades con característica de secuencialidad de manera preferente, facilitando al estudiante en una fase inicial poder aproximarse a un panorama general de las tareas y las temáticas, es decir de los contenidos y las acciones de 
aprendizaje, para luego introducirlo en un paso a paso de las acciones, aportes, tareas y productos a desarrollar en el curso.

Acorde a los resultados del estudio se debe resignificar el valor de la experiencia, reconociendo que los sujetos tienen vivencias propias que hacen parte de los saberes que construyen. A su vez, lo significativo cobra validez especial en la educación en ambientes virtuales de aprendizaje cuando se identifica que cada estudiante tiene un contexto propio en el cual construye saberes con sentido dialógico. De esta manera se debe generar una renovación permanente del contenido de los cursos de primera matricula, de las acciones de mejora a incorporar en los cursos, para responder de manera efectiva al proceso formativo de los estudiantes.

Las tendencias encontradas acorde al modelo de Felder y Silverman (1988), y su análisis permitirá que se proyecten, propongan y diseñen actividades y recursos educativos obedeciendo a las condiciones particulares de los estudiantes de primer ingreso, en las que se privilegie acceder y comprender la información más desde lo visual, sensorial, secuencial y activo que desde lo verbal, reflexivo, global e intuitivo; por ello debe reforzarse la utilización de recursos con alto contenido visual y auditivo, así como precisar una instrucción detallada y simple de indicaciones paso a paso que permita la realización de tareas y la adaptación al modelo de aprendizaje.

Las tendencias en los estilos de aprendizaje se convierten en un indicador para aportar a la reflexión del cómo aprenden los estudiantes cuando inician su proceso formativo en la modalidad virtual y hacia dónde deben enfocarse los modelos de enseñanza para favorecer y enriquecer dichos estilos; se debe propiciar el desarrollo de contenidos y actividades donde se provean nuevas formas de acercarse al aprendizaje.
Los estudiantes se sienten más cómodos frente a los cursos donde se privilegian sus estilos de aprendizaje, más felices con relación a los contenidos de estudio cuando se utilizan recursos que los motivan e invitan a comprometerse con las actividades propuestas en el curso, actividades lúdicas con recursos variados para la comprensión de los contenidos; todo ello basado en los estilos, tendencias y formas de aprender de los estudiantes.

El valor de la identificación de los estilos de aprendizaje de los estudiantes y su influencia en la formación académica misma, cobra importancia entre otras razones, al permitir generar otras formas de pensamiento (Troyano, Breitman y Gete-Alonso, 2004) e ir desarrollando habilidades y estrategias en los estudiantes frente a los nuevos conocimientos y contenidos de aprendizaje, en ese sentido sería interesante en futuros ejercicios investigativos analizar si hay fluctuaciones, cambios, modificaciones o varianzas en los estilos de aprendizaje de los estudiantes de últimos periodos, con relación a las tendencias de éstos en su ingreso a la universidad.

El planteamiento de propuestas pedagógicas para favorecer los procesos de aprendizaje a partir de los estilos predominantes en los estudiantes, se convierte en una alternativa interesante y útil para mejorar la práctica pedagógica de los tutores, ya que su estructura permite de una manera sistemática avanzar en la generación de acciones específicas que faciliten los procesos de aprendizaje teniendo en cuenta los estilos cognitivos detectados, a su vez estas propuestas pueden maximizar la articulación entre la construcción de actividades, recursos educativos e indicadores de logro, para beneficiar a los estudiantes en el proceso de adaptación a la metodología, comprensión y aprobación de los cursos académicos. 


\section{REFERENCIAS}

Aguirre, M., Cancino M. O. y Loaiza N. (2009). Estilos de aprendizaje: una propuesta didáctica para la enseñanza de lenguas extranjeras. Cali: Universidad del Valle,

Alonso, C. M., Gallego, D. J. y Honey, P. (1994). Los estilos de aprendizaje. Procedimientos de diagnóstico y mejora. Bilbao: Mensajero.

Aragón, M. y Jiménez, Y. (2009). Diagnóstico de los estilos de aprendizaje en los estudiantes: Estrategia docente para elevar la calidad educativa. $C P U$-ea, (9), $1-21$.

Camarero, F., del Buey, F. M., \& Herrero, J. (2000). Estilos y estrategias de aprendizaje en estudiantes universitarios. Psicothema, 12(4), 615-622.

Cazau, P. (2001). Estilos de aprendizaje. [Online]. Recuperado de https://vdocuments.mx/estilos-de-aprendizaje-pablocazau-bueno.html

Chavero, B. (1999). Hipermedia en Educación. El modo escritor como catalizador del proceso enseñanza-aprendizaje en la enseñanza secundaria obligatoria. [Tesis Doctoral]. Universidad de Extremadura, Instituto de Ciencias de la Educación, Badajoz, España.

Chevrier, J., Fortin, G., Théberge, M. \& Le Blanc, R. (2000). Le LSQ-Fa: une version française abrégée de l'instrument de mesure des styles d'apprentissage de Honey et Mumford. Le style d'apprentissage, 28(1), 1-137. Recuperado de https://www.acelf.ca/c/revue/ revuehtml/28-1/07-chevrier.html

Curry, L. (1987). Integrating concepts of cognitive or learning style: $A$ review with attention to psychometric standards. Otawwa: Canadian College of Health Service Executives.
Díaz-Barriga, F. y Hernández G. (2003). Estrategias docentes para un aprendizaje significativo: una interpretación constructivista, 2 ed. México, D.F.: McGraw Hill.

Dunn, R., Dunn, K. \& Price, G. (1985). Manual: Learning Style Inventory. Lawrence: Price Systems.

Durán, E. y Costaguta, R. (2007). Minería de datos para descubrir estilos de aprendizaje. Revista Iberoamericana de Educación, 42(2), 1-10.

Felder, R. y Brent, (1996). Matters of Style. ASEE Prism, 6(4), 18-23.

Felder, R. M. \& Silverman, L. K. (1988). Learning and Teaching Styles in Engineering Education Application. Journal of Engineering Education, 78(7), 674-681.

Felder, R. y Spurlin, J. (2005). Applications, Reliability, and Validity of the Index of Learning Styles. International Journal of Engineering Education, 21(1), 103-112.

Ferrel, F., Thomas, J., Solano, A., Redondo, E. y Ferrel, L. (2014). Bienestar psicológico en profesores de una Universidad privada del distrito de Santa Marta. Cultura Educación y Sociedad, 5(2), 61-76. Recuperado de https://revistascientificas.cuc.edu.co/ culturaeducacionysociedad/article/ view/885

García, J. Santizo, J. y Alonso, C. (2009). Uso de las TIC de acuerdo a los estilos de aprendizaje de docentes y discentes. Revista Iberoamericana de Educación, 48(2), 1-14.

Garza, R. y Leventhal, S. (2000). Aprender cómo Aprender. México, D.F.: Trillas.

Grasha, A. F. \& Riechmann, S. W. (1975). Student learning styles questionnaire. Cincinatti: University of Cincinatti. 
Hernández, R. Fernández, C. y Baptista, M. (2010). Metodología de la Investigación. México, D.F.: Mc Graw Hill.

Jensen, E. (1994). Unlocking The Code: Learning Styles. Brain Based Learning and teaching. Beirut: Turning Point Publisher.

Johnson, R. \& Onwuegbuzie, A. (2004). Mixed method research: A research paradigm whose time hascome. Educational Researcher, 33(7), 14-26. https:// doi.org/10.3102/0013189X033007014

Keefe, J. (1988). Aprendiendo Perfiles de Aprendizaje: manual de examinador. Reston: Asociacion Nacional de Principal de Escuela de Secundaria.

Kolb, D. A. (1984). Experiential learning: experience as the source of learning and development. New York: Prentice hall.

Litwin, E. (1997). Las configuraciones didácticas. Buenos Aires: Paidos.

Mc Carthy, B. (1987). El Sistema MATE: enseñando a aprender tren con métodos de izquierda derecha técnica. 2 ed. Barrington: Superar.

Pearson, R. (2007). Estilos de aprendizaje. [Online]. Recuperado de https://www. jelaprendizaje.org/

Pérez, J. (2001). Programación Neurolingüística y sus estilos de aprendizaje. [Online]. Recuperado de http://www. aldeaeducativa.com

Revilla, D. (1998). Estilos de aprendizaje. Temas de Educación. [Segundo Seminario Virtual]. Lima: Pontificia Universidad Católica del Perú.

Rodríguez, J., G. Fajado, F. Higuera y González, J. (2006). Estilos de aprendizaje en internos de pregrado. Revista Hospital General Dr. M. Gea González, 7(3), 102-107.
Roset, T. B. (2003). Los estilos de aprendizaje. [Online]. Recuperado de http:// www.monografias.com/trabajos12/losestils/losestils

Senior, A., Colina, J., Marín, F., Perozo, B. (2012). Visión complementaria entre los métodos cualitativos y cuantitativos en la investigación social. Una aproximación teórica. Muticiencias, 12(Extraordinario), 106-114.

Troyano, H., Breitman, M. y Gete-Alonso, C. (2004). Estilos de aprendizaje que predominan entre los estudiantes universitarios. Revista de enseñanza universitaria, (23), 63-82.

Valadez, M. (2009). Estilos de aprendizaje $\mathrm{y}$ estilos de pensamiento: precisiones conceptuales. Revista de Educación y Desarrollo, (11), 19-30.

Wenden, A. L. (1991). Learner strategies for learner autonomy: Planning and implementing learner training for language learners. Hertfordshire: Prentice-Hall International.

Willing, K. (1988). Learning Strategies as information management: some definitions for theory of learning strategies. Prospect, 2(3), 139-155.

Witkin, H. (1985). Estilos Cognoscitivos naturaleza y orígenes. Madrid: Pirámide.

Woolfolk, A. (1996). Psicología Educativa. México, D.F.: Pearson. 